\title{
Ethnic flows in the Tibetan-Yi corridor throughout history
}

\author{
Shuo Shi
}

Received: 12 April 2018 / Accepted: 25 April 2018 / Published online: 08 June 2018 (C) The Author(s). 2018 Open Access This article is distributed under the terms of the Creative Commons Attribution 4.0 International License (http://creativecommons.org/licenses/by/4.0/), which permits unrestricted use, distribution, and reproduction in any medium, provided you give appropriate credit to the original author(s) and the source, provide a link to the Creative Commons license, and indicate if changes were made.

\begin{abstract}
Fei Xiaotong not only put forward the concept of the Tibetan-Yi Corridor, but also pointed out that it was an "ethnic area shaped by history." How did the corridor form throughout history? After studying the ethnic flows of the Tibetan-Yi Corridor in history, this paper points out that there were mainly five trends in the area: 1. The Tibeto-Burman ethnic group migrating from north to south; 2. The Tubo eastward expansion and the process of "Fan-isation" in the north of this corridor; 3. The Mongolian southward migration; 4. the Mu chieftains and Yi northward expansion in the Ming and Qing Dynasties; 5. The Han pouring into the area in the Ming and Qing Dynasties until the Republic of China. Analyzing these trends, the author believes that the ethnic flows of these five large ethnicities have fundamentally shaped today's ethnic patterns in the Tibetan-Yi Corridor. The study may help us understand the corridor as an "ethnic area shaped by history."
\end{abstract}

Keywords Tibetan-Yi Corridor · Tibeto-Burman ethnic group $\cdot$ Ethnic flow

\section{Introduction}

Since 2000, the Tibetan-Yi Corridor has gradually become a key region for Chinese ethnological, ethno-historical, and anthropological research. As the area with the largest number and most complex systems and branches of ethnicities in China, and the best-preserved proto-forms of ethnic cultures, the cultural diversity, uniqueness and complexity of the Tibetan-Yi Corridor are also being increasingly valued by ethnologists. It

S. Shi $(\square)$

School of History\&Cultures, Sichuan University, Chengdu 610064, China

e-mail: lindalina126@163.com 
should be noted that in the 1980s Fei Xiaotong had put forth the concept of "Tibetan-Yi Corridor," with the important precondition that he regarded it an independent "ethnic region shaped by history," and he gave the following description of the historical features of the Tibetan-Yi Corridor: "This corridor was the bordering area of contact between the Han and the Tibetans, between the Yi and the Tibetans, and in different historical phases it presented a situation of political seesawing. This corridor was the area where nationalities called Qiang, Di, Rong, etc., lived, and where local regimes of different sizes and longevity appeared."(Fei Xiaotong,1980) also pointed out that: "We ought to further clarify the process of ethnic evolution of this entire corridor."(Fei Xiaotong,1980) "If we could thoroughly describe this corridor, we would be able to answer a great number of questions about ethnic formation, contacts, amalgamation, changes, and so on "(Xiaotong Fei,1982). So, as an "ethnic region shaped by history," what kind of process of "historical formation" did the Tibetan-Yi Corridor undergo? In other words, how did the current ethnic features and patterns of the Tibetan-Yi Corridor come into place? This paper will attempt to carry out a preliminary study on the ethnic flows in the Tibetan-Yi Corridor throughout history from a macroscopic perspective, in order to contribute to the understanding and comprehension of it as an "ethnic region shaped by history."

The reason why the Tibetan-Yi Corridor is called a "corridor" is because it is located in the area of the Hengduan mountains, with mountain ranges and rivers going north to south, geographically forming a typical "corridor" shape, and thus becoming a natural passage. Migrations and flows became the most significant trends in the history of the ethnic activities in this "corridor"-shaped geographical region. Even though the ethnic migrations and flows in this area have been frequent and complex throughout history, generally speaking, historically the ethnic flows in the Tibetan-Yi Corridor have mainly presented five trends, which we are going to describe in the following sections.

\section{Methods}

This study based on reading a lot of historical documents and doing plenty of field work.

\section{Results and discussion}

The migration of the Tibeto-Burman ethnic group from north to south

In terms of ethnic features, the Tibetan-Yi corridor is mainly populated by the Tibeto-Burman ethnic group. ${ }^{2}$ The sixteen ethnic groups currently distributed in the

\footnotetext{
${ }^{1}$ Fei Xiaotong divided the areas where the Chinese people live into six "historically shaped regions," namely: the northern grasslands region, the north-eastern mountainous forest region, the south-eastern Qinghai-Tibetan plateau, the Tibetan-Yi corridor, the Yunnan-Guizhou plateau, the Nanling corridor, the coastal region, and the central plains. See FeiXiaotong, "On the Issue of Ethnic Classification in China," Social Sciences of China, 1980 (1).
} 
Tibetan-Yi Corridor, namely the Tibetans, Qiang, Yi, Bai, Nakhi, Lisu, Pumi, Dulong, Nu, Achang, Jingpo, Lahu, Hani, Jinuo, Menba, and Luoba, all belong to the Tibeto-Burman ethnicity. The Tibetan-Yi Corridor is the main area inhabited by the Tibeto-Burman ethnic group, as well as an important historical area of its development and differentiation. In the past the academic world would link the origins of the Tibeto-Burman ethnic group with historical "Di and Qiang people," believing the ancient $\mathrm{Di}$ and Qiang people to be the main source of today's Tibeto-Burman group; it was also generally accepted that the Di and Qiang people came from the upper reaches of the Yellow River in the Gansu-Qinghai area. Even though the fact that the primitive ancestors of the Tibeto-Burman ethnic group were collectively called "Di and Qiang people" is still worth discussing, (Science Press, 2002) archeological data prove that the earliest inhabitants of the Tibetan-Yi Corridor undeniably came from the upper reaches of the Yellow River. The primitive inhabitants of the upper reaches of the Yellow River must have migrated south into the Tibetan-Yi Corridor, during the middle or late Neolithic Era, circa 6000 or 5500 BCE. In the present age three of the earliest Neolithic relics were discovered in the Tibetan-Yi Corridor, namely the Yingpanshan ruins at the upper reaches of the Minjiang River, the DanbaHan'e ruins at the upper reaches of the Dadu River, and the Kham Karuo ruins at the upper reaches of the Lancang River, dating back to 5000-5500 BCE, the earliest Neolithic ruins discovered so far in the Tibetan-Yi Corridor. Not only are these three sites distributed in the northern part of the Tibetan-Yi Corridor, but they also clearly show identical cultural traits with the cultures of the upper reaches of the Yellow River, presenting painted pottery, millet and tools typical of the upper reaches of the Yellow River (Shuo Shi, 2006). The Neolithic cultural features of the Tibetan-Yi Corridor also displayed two clear-cut traits: firstly, the ruins in the areas closer to the upper reaches of the Yellow River are the oldest ones, while the ones farther away from the upper reaches of Yellow River are more recent; secondly, the closer the ruins are to the Gansu-Qinghai area, the more distinct are their cultural features, while the farther they are, the weaker these traits get (Shuo Shi, 2006). This explains why both archaeological and cultural traits clearly show the Neolithic cultures of the upper reaches of the Yellow River following gradual trends of propagation and development towards the Tibetan-Yi Corridor region (Cultural Relics Press, 1985). This definitely indicates that the earliest residents of the Tibetan-Yi Corridor came from the area of the upper reaches of the Yellow River, and it shows that during the Neolithic Era the inhabitants of the upper reaches of the Yellow River experienced large-scale migration waves towards the Tibetan-Yi Corridor. Paleo climatology studies indicate that

\footnotetext{
${ }^{2}$ Fei Xiaotong called it "Tibetan-Yi Corridor" because nowadays the Tibetan and Yi people are the two most populous ethnic groups in the corridor, occupying the widest area, and they were thus used as reference. However, with regards to the ethnic connotation of the Tibetan-Yi Corridor, in 1981 Ma Yao used the term "the corridor of the Tibeto-Burman ethnic group" in a speech delivered at the opening of the Institute of Chinese South-Western Ethnicities. Although this nomenclature may not be as aesthetically pleasing and widespread as "Tibetan-Yi Corridor," it reflects more accurately the ethnic connotation of this corridor. See Ma Yao, "Review and Prospective of the Study of Chinese South-Western Ethnicities," edited by the Institute of Chinese South-Western Ethnicities, South-Western Ethnicities Studies, Sichuan Ethnicities Publishing House, 1983, p.16.
} 
around $6000 \mathrm{BCE}$ the climate of the Yellow River basin rapidly became colder and drier, which was probably the main reason causing the original inhabitants of the upper reaches of the Yellow River to experience a large-scale southward migration.(Li Wenyi, 1998) Historical linguistics studies suggest the origins of the Tibeto-Burman ethnic group and its separation with the Chinese language certainly happened in the upper reaches of the Yellow River. (Wu Anqi, 2002) These Neolithic inhabitants who migrated southward to the Tibetan-Yi Corridor about 6000 years ago were the main pioneers of the Tibetan-Yi Corridor, as well as the earliest ancestors of the Tibeto-Burman ethnic group (Shuo Shi, 2008).

The primitive inhabitants who migrated southward from the upper reaches of the Yellow River to the Tibetan-Yi Corridor first established a number of relatively large settlements by the upper reaches of the Lancang, Dadu, and Minjiang rivers, in the northern part of the corridor, forming a rather mature and prosperous Neolithic culture. Soon after that, a portion of the population migrated south along the river valley of the Tibetan-Yi Corridor, arriving in the southern part of the corridor circa $4000 \mathrm{BCE}$, and establishing a certain degree of exchanges and relations with the primitive population of the Yangtze river basin and the northern part of Southeast Asia in southwestern and central Yunnan. ${ }^{3}$ This proves that in the late Neolithic Era the ancestors of the Tibeto-Burman people had moved south in the region of western Yunnan and northern Vietnam, covering the majority of the Tibetan-Yi Corridor, and becoming the main population system in the area. Since the ancestors of the Tibeto-Burman people migrated south during the Neolithic Era at different times and chose completely different passages and routes, (Shuo Shi, 2009a, 2009b, 2009c) throughout the migration process they became gradually differentiated in terms of cultural features, starting to shape different population systems and branches.

Furthermore, the migrations of the Tibeto-Burman people included the southward migration of the Shu people. After the Qin destroyed the Shu in $316 \mathrm{AD}$ with the mass migration of Qin people into the territory of the Shu, ${ }^{4}$ leading the surviving forces of the Shu royal family and its people to initiate a large-scale southward migration. The Shu people mostly moved south through Xi, and arrived in the modern day border area between Sichuan and Yunnan, in the southern part of the Tibetan-Yi Corridor, asthe Records of the Grand Historian: Table of the Three Ages, Justice cites in this sentence, which was recorded in the Puji: "After the kingdom (of Shu) was destroyed, their descendants moved far away and settled down in Yao, Xi and et cetera." According to historical records, approximately during the later stage of the Warring States era up until the early

\footnotetext{
${ }^{3}$ From archaeological evidencewe can see that the Neolithic cultural systems originating from the upper reaches of the Yellow River came in contact with two other Neolithic cultures in the southern part of the Tibetan-Yi Corridor: one was the cultural system of the single or double stone axe and the section axe, which were closely related tocoastal Neolithic cultures in Southeastern China; the other one was the flat stone axe and hatchet systems, and the blade hatchet system (boot-shaped hatchet), closely connected to Neolithic cultures in northern Vietnam. See Wang Dadao, "Rethinking the Classification of Neolithic Cultures in Yunnan,"Tibetan Archaeology, Ser. 1, Sichuan University Press, 1994, p.104.

${ }^{4}$ The Chronicles of Huayang: Records of Shu, the Records of the Grand Historian: Annals of Xiangyu, and the Book of Han: Annals of Emperor Gaozu all contain records of the large-scale migration of the Qin to Shu territories.
} 
Western Han dynasty, the self-proclaimed "Anyang king,"that is the Shu prince, led thirty thousand soldiers to northern Jiaochi, (a southernmost province of the Chinese Empire), where he established a new regime (Zhonghua Book Company, 1978). The Records of the Great Historian: Table of the Three Ages also notes that: "The kings of Shu, [...] traveled frequently for five thousandli from the South-West of the Han dynasties to present tributes to the Han." As a matter of fact, in the historical records from the Han, Wei and Jin dynasties, the names of peoples like the $\mathrm{Xi}$ and the Sou we find in the records about the barbarians ( $\mathrm{Yi}$ people) from the southwest are all connected to the Shu people migrating south (Shuo Shi, 2009a, 2009b, 2009c, 2011). Later these Shu people integrated into the Yi sub-group living in the southern part of the Tibetan-Yi Corridor.

In short, the migration of the Tibeto-Burman people from north to south was the most important population flow trend in the Tibetan-Yi Corridor prior to the Tang dynasty, basically establishing the distribution pattern of the Tibeto-Burman group within the Corridor. This point is also amply confirmed by ethnographic data. The Tibeto-Burman people currently living in the corridor clearly retain the historical memory that their ancestors came from "the North" in their epics, legends, and cultural customs. For example, the Qiang people living by the upper reaches of the Minjiang River say in their legends that their ancestors came from the Gansu-Qinghai region, (Changshou Ma, 1984) while the legends of the Yi people and the ancient records written in Yi language claim that their ancestors were originally from "outside the land of the yaks" (maoniujiaowai, today's plateau region in Sichuan), ${ }^{5}$ and according to Nakhi legends, their ancestors came from the upper reaches of the Jinsha River; the Lisu people say that their ancestors came from the upper reaches of the Lancang River, and the Jingpo people say their ancestors came from an unspecified area of the Qinghai-Tibetan plateau. (Meng Mo, 1980) Today the tradition of "escorting the soul" is still very common in the funerary customs of the Yi sub-group living in the southern part of the Tibetan-Yi Corridor: this practice consists of inviting a priest after somebody dies (each ethnic group has different names for priests: the Yi people call them bimo, the Nakhi people call them dongba, and so on), to read the sacred book for escorting the soul ofthe deceased, in order to help their soul findthe land of their ancestors, one station after another (Shuo Shi, 2001). Even though the paths and the toponyms along the routes of the "escorting the souls" of each nationality are completely different, all of them are directed "northward." The "escorting the souls" traditions of the Yi, Nakhi, Hani, Lisu, Lahu, Jinuo, Pumi, and Jingpo people are the most representative and prominent.(Yunnan Bureau for the Plan of the Publication of Nation, 1989; Ling Fuxiang, 1993) This is a real reflection of the history of the migration from north to south of the ancestors of the Tibeto-Burman group. Just as ethnologist Li Shaoming said: "Each group of the Tibeto-Burman people living in the

\footnotetext{
${ }^{5}$ Vol. 22 Chronicles of Puliof the Anshun Government Records, Guizhou, cites the ancient record in Yi language Yi Book of Luogui, according to which "Mengzhe, the founder [the ancestors of the Yi people] moved away from outside the land of the yaks and settled in Qiongzhilu.'Brief History of the DebuPeople, (duplicate of the translation), translated by the GuizhouBijie Prefecture Yi Language Translation Unit. See also Meng Mo, "On the Question of the Origins of the Yi people, "The Ideological Front, 1980 (1).
} 
valleys of the six rivers of the Tibetan-Yi Corridor share a history of migration from north to south" (Shuo Shi 2009a, 2009b, 2009c).

The eastward expansion of Tubo and the "fan-isation" of the northern part of the Tibetan-Yi corridor

Between the 7th and ninth century CE, the Tubo dynasty, which arose abruptly in the Tibetan plateau, initiated a strong expansion towards the Tibetan-Yi Corridor. In the late seventh century, not only did the Tubo incorporate the Nanzhao kingdom as a subordinate tribe, but also "incorporated the territory of the Yangtong, Tangut and various Qiang tribes, bordering the territories of the Liang, Song, Mao and Xi to the East," (Shaoming Li 1994) and their influence extended to today's region of the upper reaches of the Minjiang and Dadu Rivers. In 763 AD the Tubo army attacked Chang'an, and after retreating from Chang'an, the Tubo troops marched south, joining the Nanzhao army, and breaching the Tang territories of $\mathrm{Xi}$, Song, Wei, and Bao. In $783 \mathrm{AD}$, the Tang and Tubo concluded the meetings in Qingshui, stipulating borders along the Minjiang and Dadu Rivers, with the Tang dynasty to the East, and the Tubo to the West. (Old Book of Tang 1975) The demarcation of these borders meant that the Tubo already occupied the entirety of the northern Tibetan-Yi Corridor.

The expansion of the Tubo towards the Tibetan-Yi Corridor had two consequences: firstly, the conquest and ruling of numerous tribes in the Tibetan-Yi Corridor, especially in the north, which became subordinate tribes; many of these conquered tribes were incorporated into the Tubo army and sent to fight external forces, becoming an organic part of the Tubo military alliance. Therefore, the conquest and ruling of the tribes of the Tibetan-Yi Corridor by the Tubo meant that numerous tribes that originally were locally scattered and non-subordinated were joined in one political and military entity; in addition, the Tubo rule lasted for over a hundred years, strongly promoting the process of integration among the local tribes. Secondly, a big part of the Tubo army entered the Tibetan-Yi Corridor. The Tubo forces were formed by different native Tubo tribes. In The TuboCommentary in the New Book of Tang we read: "According to the laws on prisoners of war, they exiled the Hao clan and theirslaves, sending them to till and pasture on their own." The Tubo troop formed by the Hao clan and their slaves was a military organization as well as a production unit, and in reality their expedition to the east also constituted an ethnic migration. These troops coming from the native land of the Tubo mixed with the subdued local tribes over a long period of time, leading to mutual exchanges and influence, which over the years led to reciprocal assimilation and fusion, thus causing the gradual trend of "Fan-isation" of the northern part of the Tibetan-Yi Corridor. This became particularly clear after the end of the Tubo dynasty. After the collapse of the Tubo regime, a civil war broke out between the descendants of the royal family fighting over the throne, and many Tubo troops and accompanying soldiers and slaves from the eastern part of the Tubo territory were unable to return to their homeland in Tubo. According to historical records, they "had nowhere to go, so they gathered a few thousand people, calling themselves 'Wenmo' and settling down between Gansu, Guazhou, Shazhou, and 
between He, Wei, Min, Kuo, Die, and Dang." (Old Book of Tang 1975) The main bulk of the so-called "Wenmo" volunteer army was composed ofTubo slaves, and it included Tangut and Tuyuhun inhabitants that were originally subsidiary tribes of the Tubo, and troops formed by the mixing of different ethnicities, including the Han. (New Book of Tang 1976) One branch of the Wenmo volunteer army spread to the Tibetan-Yi Corridor, and they were recruited by Gaopian, the provincial governor of Jiannan, and were led south by the Wenmo chief Lu Ruyue, arriving in the Dadu River basin, which they garrisoned, helping the defense of the Nanzhao kingdom; later on they gradually scattered over western Sichuan and the plateau in north-western Yunnan, where they mixed with the local tribes, entering a path of interdependency and integration. This trend slowly continued and developed among the people, resulting in gradual changes to the ethnic features of the northern part of Tibetan-Yi Corridor. The History of Liao records that at that time Liao sent envoys to the original separatist forces in Qinghai-Tibet, the latter called "great Fan," "minor Fan," "Tubo," "Western Fan," and so on (Wenzhou Zhou 1982). The so-called "descendants of the Tubo" by the Song people were mainly distributed in Gansu, Qinghai and the Western Sichuan plateau in the northern part of the Tibetan-Yi Corridor, which were precisely the territories that were conquered and ruled by the Tubo for so many years. The Song collectively named the population of the aforementioned territories "descendants of the Tubo," which indicated that at the time the cultural traits of those regions displayed profoundly "Fan" features. It is necessary to note that before the Tang dynasty, historical records also called the people living in the Tibetan-Yi Corridor "Yi,"“Qiang,"“Di," or "Yue," while the names "Bo" or "Fan" were absent; the new population named "Bo" (or "Fan") appeared after the Tang dynasty. After the Song and Yuan dynasties, the term "Bo" was gradually replaced by the name "Fan." During the Ming and Qing dynasties, up until the Republic of China, "Fan" finally became a term of ethnic classification extensively used in the Tibetan-Yi Corridor, especially the region of the Western Sichuan plateau, as indicated in the Han historical records. Undoubtedly, the emergence of the new ethnic classification of "Bo" or "Fan" is the result of the conquest and assimilation of the tribes in the Tibetan-Yi Corridor by the Tubo dynasty between the 7th and ninth century, as well as the result of a large quantity of Tubo soldiers and slaves flowing into those territories, and gradually mixing with the local tribes over a long period of time, gradually integrating with them after the fall of the Tubo. The appearance of "Bo" or "Fan" reflected the fact that starting from the Song dynasty the ethnic features of the northern part of the Tibetan-Yi Corridor started to change, and ethnic amalgamation reached a new level.

During the Song dynasty, the northern part of the Tibetan-Yi Corridor was referred to with the new ethnic categories "Bo" or "Fan," and there was an important backdrop: after the eleventh century AD, Tibetan Buddhism gradually moved from west to east, achieving wide dissemination and development within the local territories, causing the local cultural features and social structure to gradually become similar to Tibet, and residents in both regions started to become uniform in terms of faith, cultural psychology and written and spoken language (since religious propagation always uses the vehicle of language). This process coincided with the process of the shaping of the Tibetan ethnic group. The essence of the formation of 
the Tibetan group was a process of incorporation of the tribes and clans of the Qinghai-Tibetan plateau, which was mainly completed through two stages: in the first one the Tubo dynasty's conquest and rule over the many tribes and clans of the Qinghai-Tibetan plateau for more than 200 years; in the second one, after the tenth century the dissemination and development of Tibetan Buddhism in the Qinghai-Tibetan plateau. If the former can be said to be a process of aggregation and integration on a political and regional level, the latter was a process of integration on a cultural level, which played a decisive role in ultimately shaping the common language, psychological qualities and culture of the peoples in all regions of the Qinghai-Tibetan plateau (History of Liao, 1987). If we look at it from the point of view of the expansion of the Tubo dynasty towards the Tibetan-Yi Corridor and the subsequent dissemination of Tibetan Buddhism in local areas, its biggest result was to lead to the "Tibetanisation" of the entire northern part of the Tibetan-Yi Corridor, namely the Western Sichuan plateau. In fact, during the Song dynasty the ethnic categories called "descendants of the Tubo,"“Bo," and later "Fan," started to gradually appear in the northern part of the Tibetan-Yi Corridor, which really indicated the later "Tibetan" (Shuo Shi 1997). Thus, the expansion of the Tubo towards the Tibetan-Yi Corridor and the following dissemination and development of Tibetan Buddhism in local areas, is the reason and driving force of the formation of the pattern of "Tibetans in the north." Even though the "Tibetanisation" of the northern part of the Tibetan-Yi Corridor was a gradual process, if we consider the fact that the Song dynasty started widely using the terms "descendants of the Tubo," "Bo" and "Fan" to refer to the population of this area, the time of the pattern formation of the northern "Tibetans" of the Tibetan-Yi Corridor is basically to be dated around the 11 th-twelfth century.

\section{The Mongolian southward migration}

In the early thirteenth century, before the Mongols abruptly rose in the north, establishing the Yuan dynasty in the Central Plain, it firstly adopted the strategy of "first taking the Bos in the South-West to pursue the whole of China."(Ren Naiqiang, 2000) Because of setbacks in the battlefield in Sichuan, in 1253, in order to "outflank the Dali Kingdom, turn around and attack the Song," Kublai Khan ordered his 100 thousand army to split into three routes and move to the south from the Tibetan-Yi Corridor, to pacify Dali, and to ensure that all tribes "would submit to their authority" along the way (History of Yuan, 1976). When Kublai Khan, as a supreme khan, founded the Yuan dynasty in 1260, the Mongolian military strength already had general control of most parts of the Tibetan-Yi Corridor: he dispatched troops there, implemented a system of local officials, established an administrative subdivision and opened relay stations, thus starting the Yuan rule over the Tibetan-Yi Corridor, which lasted for almost a century.

After the collapse of the Yuan dynasty, a great part of the Mongolian forces withdrew northward, and the remaining Mongol troops continued to have a relatively big influence on the Tibetan-Yi Corridor, where, in the early Ming dynasty, the surrendered Mongolian general Yuelutiemu'er started a rebellion in Jianchang, which lasted for seven or 8 years (Wenhai Cheng 1986). Beginning from the mid- 
tolate Ming dynasty, following the relaxation of the Ming policy of "isolation of the Mongols and Fan people," some Mongolian tribes migrated down south to Qinghai in succession in search of pastures: some of these tribes started to enter the Tibetan-Yi Corridor successively and sporadically. This trend gradually accumulated and developed until the late Ming and early Qing dynasties, because of the wars in the Central Plain and the invoking of the new Gelugpa power in Tibetan territories, ultimately leading the Mongolian Heshuote tribe in Qinghai to undertake a large-scale southward migration to the Tibetan-Yi Corridor; they defeated the enemy of the Gelugpa Qianghaobaili headman in the northern part, and afterwards they continued advancing southward, controlling the territories of present day's Dartsendo, Litang, Batang, Muli, and Draba, (History of Ming, 1977) Wu Sangui, who was defending Yunnan, "ceded to" the Mongols the territories on both sides of the Jinsha River originally administered by Muchieftains, (Xinyu Zhao 2002) and in this way the Heshoute Mongols occupied the entire northern part of the Tibetan-Yi Corridor, once again imposing Mongolian rule on this territory. After the Heshoute Mongols took control of the Tibetan-Yi Corridor, they mainly treated it as a territory to levy taxes, and they dispatched officials in every part of it to impose taxation, in order to supply the nomadic troops in Qinghai. This situation continued for several decades. Since Güshi Khan sent troops southward to the Tibetan-Yi Corridor in 1639, until the 2nd year of the reign of Yongzheng (1724), when the Qing court pacified the armed rebellion led by the chief of the Heshoute Mongols, Lob Tsangdanjin, ending their control over the Tibetan-Yi Corridor, the Heshoute Mongols ruled over the northern part of the Tibetan-Yi Corridor for approximately 80 years, and greatly influenced the local ethnic structure.

During the Yuan dynasty, as well as the late Ming and early Qing, the Mongolian forces twice undertook a large-scale southward migration to the Tibetan-Yi Corridor, ruling over the territory each time. Since the Mongolian regime in this region lasted for about two centuries, it created close relations between the Mongolian ethnic group and the nationalities living in the Tibetan-Yi Corridor. It is particularly important to note two points: firstly, many Mongols entered the Corridor. We cannot obtain statistics from the Yuan dynasty, however, we know that during the late Ming and early Qing the number of Mongols moving to the Tibetan-Yi Corridor was rather impressive, and that they were widely distributed across the territory. According to late Qing and Republican studies, the Mongol population in Xikang had an extremely wide distribution in nearly every county (Tuiji Ni 1986). In ethnic surveys conducted in the 1950s, the Mongolian population of the Tibetan-Yi Corridor was mainly distributed in two areas: one was the two counties of Yanyuan and Muli in south-western Sichuan; the other was Luding county in Sichuan. (Yang Zhonghua, 1937) We can see that during the late Qing and Republican period the Mongolian population in the northern part of the Tibetan-Yi Corridor, namely the Western Sichuan plateau, was also considerably extensive. Secondly, in later years a great number of the Mongolians in the Tibetan-Yi Corridor gradually integrated into the local ethnic groups, especially the local Tibetans in the northern part of the Corridor, becoming a new ethnic component in that territory. 
The Mongolian migration to the Tibetan-Yi Corridor caused a relatively great shock and suffering to the local ethnicities, however it also opened up and revitalized the territory, allowing it to form close relations with the political structure of the entirety of China. It can be said that the two Mongolian migrations and the close integration between Mongols and Tibetans in the Tibetan-Yi Corridor, were organic components of the integration between the two great Mongolian and Tibetan ethnic groups after the thirteenth century. The integration and amalgamation between the Mongols and Tibetans strengthened the power of the Tibetans living in the corridor, while also expanding the geographical spread of the "Tibetans in the north."

The mu chieftains and the Yi northward expansion in the Ming and Qing dynasties

During the Ming and Qing dynasties, while Mongolian forces were migrating southward in succession, a northward ethnic flow took place in the Tibetan-Yi Corridor: it was the northward expansion of the Mu chieftains and Yi people.The ancestors of the Nakhi people were called "Mosha,"“Mosuo," "Maxie," or "Moxie," and were primarily distributed in the Jinsha River valley, in present day Lijiang. Before the Yuan dynasty, the Moxie tribes were rather weak, while during the Yuan dynasty the Mu clan of the Moxie received "preferential treatment" by the Yuan dynasty, because they spontaneously submitted to them: they were appointed tea officials, general military and civilian governors and military and civilian advisors of Lijiang, (Jiaming Yang, 1997) becoming more and more powerful. During the reign of Hongwu of the Ming dynasty, the prefect of Lijiang, Ajia'ade, spontaneously "submitted" to the Ming dynasty, and the emperor Taizu granted him the family name "Mu": from that moment, the Mu clan had a Han family name. Afterwards, the heads of the $\mathrm{Mu}$ clan were appointed as local prefects of Lijiang for many generations, which became a hereditary office. Under the vigorous support of the Ming dynasty, the power of the Mu chieftains rapidly ascended and it started to forcefully expand northward.

The northward power expansion of the Mu chieftains from the final years of Ming emperor Tianshun continued until the reign of the Chongzhen, lasting over 180 years and requiring hundreds of military deployments. The expansion mainly occurred in two stages: during the first phase they repeatedly sent troops, gaining control of modern day Gyeltang, Muli and Weixi; during the second one, on the basis of the consolidation of the above-mentioned occupied territories, they continued advancing northward, and substantially pushedtheir forces forward up to the Tibetan region consisting of present day Dechen (Yunnan), Batang (Sichuan) and Markam (Tibet) (History of Yuan, 1976, 1976b). According to historical records, "The Fan people in the north of Benzilan were so afraid that they surrendered to the $\mathrm{Mu}$ troops. And the $\mathrm{Mu}$ troops were all over the places fromNakhi and Gyeltang, to Batang, Litang, which are currently attached to Sichuan."6

The main cause of the rise to power and northward expansion of the Mu clan, other than its own development demands, was to obtain the help of the Ming dynasty. Starting from the early Ming dynasty, in order to deter the revival of Mongolian power, in the early stages of the Ming dynasty there had been the national 
policy of "isolating the Mongols and Fan people" and "garrisoning the Bo and Tartar people". In the early Ming dynasty, the territories of the $\mathrm{Mu}$ clan were often plundered and weakened by the "Fan tribal chiefs" in the north, (Qingyuan Yu, 1994) and in order to stop the southward advancement of the Tibetan forces, the Ming adopted the policy of assisting the Mu clan in resisting the southward migration of the "western Fan". 7 The inscribed board "North-South Barriers against the Fan" bestowed by the Ming court to the Mu chieftains was a full proof. The strengthening and northward expansion of the power of the $\mathrm{Mu}$ chieftains not only suited the Ming's political objective with regards to the western Fan of "dividing their forces and weakening their strength, avoiding a foreign invasion," but it also embodied the Ming policy of "using non-Han people to govern non-Han people" in northwestern Yunnan. Therefore, during the entire process of expansion of the Mu chieftains from the early Ming dynasty, they were awarded additional titles by the Ming court, with the recorded awards and titles totaling 28, and the Ming emperors also granted them a golden girdle for their "sincere service" decorated with the emperor's personal calligraphy, and made a gift of the inscribed boards detailing "north-west barrier" and "bringing peace to the frontier" (Fasheng Pan 1999). At the same time, the Mu chieftains were deferential towards the imperial court, helped them with punitive expeditions and they went to the capital to present tributes, gradually becoming the most important local separatist power between Yunnan and Tibet.

The northward expansion of the Mu chieftains led to the close mixing between the Nakhi and Tibetans: not only did it promote the dissemination of Tibetan Buddhism, specifically the Gemageju sect, to the Nakhi territories in Lijiang, but also it caused the Nakhi and their Lijiang territories to start becoming a major component and hub of the Yunnan-Tibetan trade which mostly consisted of Yunnan tea being sold to Tibet; at the same time, it facilitated to a considerable degree the amalgamation between the Nakhi and the Tibetans. In a certain sense, the northward expansion of the $\mathrm{Mu}$ chieftains brought about close relations between the Nakhi and the Tibetans, causing the Nakhi to gradually become an important binding link and bridge between the Tibetan people and the nationalities living in western Yunnan, especially the Yi sub-group.

After the northward expansion of the Mu chieftains, from the mid-Qing until the Republic of China, the Tibetan-Yi Corridor also saw the northward migration of the Yi people. Since the mid-Qing dynasty, as a result of the large-scale invasion of the Liangshan Yi territories by the Qing, as well as the rebellion of the Black Yi against local chieftains, a great number of Liangshan Yi migrated north. They either followed the river region of Anning, entering the district of Mianning and Jiulong, or moved along the Dadu River, arriving in present day Hanyuan county in Yan'an prefecture, and Luding county in Garze prefecture, or they headed north-west, arriving in great numbers in today's Muli Tibetan autonomous

\footnotetext{
${ }^{6}$ According to statistics from the List of Officials of the Mu Clan, after the formation of the Moxie Mu clan system, they sent troops to the Tubo territories bordering Yunnan, Sichuan and Tibet 103 times.

${ }^{7}$ The List of Officials of the Mu Clan records that: "In the 16th year of the reign of Hongwu, the great tribal chief of the western Fan, general Dayoubo,led a rebellion army and invaded the territory of his own governor BaiLangcang," and "in the 6th year, the territory of Baoshanzhoubai (Sanbabaidi) was plundered by the Fan traitor.
} 
county in Liangshan prefecture. The ethnic identification survey performed in the 1950s about the northward migration of the Yi people during the Qing dynasty and the Republic of China era in Selected Works on Investigation Data and Archive Material on the History of the Yi People in Sichuan is a rather centralized reflection.(List of Officials of the Mu Clan in Lijiang 1983). According to Fang Guoyu's statistics: "The 200,000 Yi who currently reside in the region of Xichang, in Sichuan, and the 70,000 Yi who live in the prefecture of Lijiang, in Yunnan, migrated from Liangshan"(Sichuan Provincial Editing Group 1987). The Brief History of the Yi People mentions that: "In the last hundreds of years, between one and two hundred thousand Yi people migrated away from Liangshan, settling in the different counties of Xichang prefecture and the mountainous area of Hanyuan and Shimian counties in Ya'an prefecture, and even Ninglang, Gyeltang, Yongsheng, Yongren, and Luquan in Yunnan" (Guoyu Fang 1984). The northward migration of the Yi ethnicgroup mainly happened in family units, and was mostly successive, scattered and progressive, causing the borders between Tibetans and Yi in the Dadu River valley and Yalong River valley in the eastern part of the Tibetan-Yi Corridor to be substantially pushed northward, and becoming a considerably large ethnic flow inside the Tibetan-Yi Corridor in recent times.

Generally speaking, the northward expansion of the Mu chieftains, together with the northward migration of the Yi people, can be regarded as a northward expansion of the Yi subgroup in the southern part of the Tibetan-Yi Corridor after the Ming and Qing dynasties, not only causing the Tibetan-Yi borders to change and be adjusted, but it also finalized the trends of the ethnic structure of the "northern Tibetans" and "Southern Yi" in the Tibetan-Yi Corridor.

The Han pouring into the Tibetan-Yi corridor during the Ming-Qing dynasties and the republic of China

The migration of a great number of Han people was an important feature of the ethnic flows in the Tibetan-Yi Corridor during the Ming-Qing dynasties until the Republican era. Although there had been Han people migrating to the upper reaches of the Minjiang River, the mid- and lower reaches of the Dadu River, and the lower reaches of the Yalong River since the Qin and Han dynasties, the Han activities in the Tibetan-Yi Corridor were mainly limited to the area east of the Dadu River (Yunnan Ethnic Minorities Socio-historical Survey Team 1963). This started to change during the Ming-Qing dynasties. After the mid-Ming, the route that crossed the Tibetan-Yi Corridor from east to west through Sichuan and Tibet started to become an important transportation road connecting the central regions and Tibet (Zhongchang Shen and Fuqua Li 1983).

In 1720 and 1727 the Qing troops twice crossed Sichuan marching towards Tibet, causing the status and significance of the path between Sichuan and Tibet to become ever more prominent. In order to ensure that the path from Sichuan to Tibet remained unobstructed, and during the reign of Qing emperor Kangxi, the Qing started setting up garrisons and provision posts along the sides of the road as well as in important mountain passes, and they appointed the Green Standard Army from Sichuan, composed of Han soldiers, for the protection of the road. This had 
two important effects: firstly, the Han officers and soldiers stationed along the Sichuan-Tibetan road gradually started to open shops, carry out trade, cultivate land, and they also began to intermarry with the local Tibetan population, causing the conversion to migrant population to a certain extent (Zhao Yi, 1992) secondly, once the Han officials and soldiers stationed in that area had brought safety and protection, an extremely diverse crowd of Han people from inland China, including merchants and officials along with their entourages, labourers, boatmen, refugees, miners, secretaries and interpreters hired by tribal headmen, started to be linearly distributed along the Sichuan-Tibetan road; among them, Han merchants were in the majority. From the reign of emperor Qianlong to emperor Jiaqing, a considerable amount of Han people entered these territories as a result of the population growth and land pressure in Sichuan, and started cultivating the land and opening mines. (Wu Feibai, 1935,)Thus, the Qing dynasty, aided by the Green Standard Army stationed along the Sichuan-Tibetan road, enabled a large-scale wave of Han people to migrate to the Tibetan-Yi Corridor.

During the late Qing and the Republican era, the quantity and scope of the Han people entering the Tibetan-Yi Corridor further increased. In 1904, the British army invaded Lhasa, and as a response to this severe border crisis, the Qing dynasty strengthened its operations by the Sichuan border, appointing Zhao Erfeng for the bureaucratization of native offices by the Sichuan border areas. Zhao Erfeng energetically put in place new policies and measures like instituting schools, opening up land for cultivation, carrying out army training, mining and trading in order to attract Han people from the inland, encouraging them to cultivate land in those regions, leading to the migration of a great number of Han people. According to historical records, at that time "the number of migrants cultivating and bundling grains over the border reached tens of thousands" (Zhenggang Liu and Weihua Tang 2002). Entering the Republican era, after Yin Changheng led an army of 5000 soldiers in a punitive expedition to the west in 1912, and the "Dabai incident" in 1930 , the $24^{\text {th }}$ army division of Liu Wenhui subsequently entered Kham because of disputes between Sichuan and Tibet. The local wars continued uninterrupted, the Han troops established garrisons in major cities and towns in Dartsendo, Batang, Litang, Garze, and Kham, and many of them settled down in those places. According to statistics, just in the first ten years of the Republic of China, the Han people who entered the Sichuan-Tibet region numbered around seven or eight hundred thousand (Shuzi Huang 1934). Afterwards, a number of factors, including the war against the Japanese, the establishment of the province of Xikang (modern day Tibet), and the resuming of Han-Tibetan trade, promoted once again a migration wave of Han people in the regions of the Tibetan-Yi Corridor. This time a more multivariate group of Han people arrived, including herb gatherers, artisans, government employees, people with odd jobs, porters, unemployed refugees, inspectors, and so on; among them, the majority were farmers, miners and merchants. According to Zhou Taixuan's General History of Xikang, "The population of Dartsendo increased after the war against the Japanese. According to a recent survey, it has surpassed 200,000 individuals. Among them, the Han people represent six or seven tenths of the population" Chongwei Chen 1930). 
It is necessary to point out that during the Qing dynasty and the Republican era, many of the Han people who migrated to the northern part of the Tibetan-Yi Corridor did not exist as an independent ethnicity, on the contrary, they rapidly blended with the Tibetan people. The main channel of mixing was intermarriage. Because during the Qing dynasty and the Republican period the Han people who arrived there were mainly officers, soldiers, merchants, farmers and artisans, they were all unmarried men, and since they lived in remote areas characterized by harsh environmental conditions, intermarriage between Han and Tibetans became a common choice for Han migrants. The Ethnic Groups of Xikang provides the following description of the intermarriages between Han and Tibetans in Xikang: "Officials, soldiers and merchants in all parts of Xikang settled down and started businesses, those who married non-Han women were myriad, and in the last thirty years the mixed-raced Xikang people (meaning the descendants of intermarriages between Han and Tibetans, called chegewa in Tibetan, which means 'half Tibetan, half Han') could be found in every city and hamlet, while entirely non-Han people could be only found in the deep of the mountains. In the late Qing dynasty, tens of thousands of soldiers and farmers all married and had children in Xikang; merchants from all parts of Sichuan and Shanxi, engaged in business in hamlets and also married non-Han women for assistance."(Taixuan) This goes to show the extensive degree of intermarriage between Han and Tibetans Xiangfeng He 1941, Zhao Liufang, 1938) The Han-Tibetan intermarriage not only led to many Han people settling down in those regions, but it also led to the deep mixing between Han and Tibetans. Because those regions were on a plateau, migrant Han had no choice but to adopt the Tibetan ways of life in order to adapt to the environment on the plateau; moreover, they married with Tibetan people, thus the ways of life of the Han people from intermarried families were Tibetanised in the course of two or three generations, becoming almost completely Tibetan. Therefore, during the Qing dynasty and the Republican era, many Han people migrated to the northern part of the Tibetan-Yi Corridor, leading to interactions between the Han and the Tibetans, with the effect of the "Han" being integrated into "Tibetans."With regard to this, Zeng Yanshu, who was stationed for a long time in Litang during the Republican period, presented the following explanations: "The Han people would often assimilate the Kang people, however, living in Xikang for a long time, marrying and having children with them, and they unconsciously started to adopt their ornaments, boots, belts, swords, language, and pelts, becoming entirely 'Kanganized' (Anonymous, 1936). When entering another environment there are requirements to be met, thus it is inevitable that the ideal loses to the fact" Yanshu Zeng 1943). However, as a matter of fact this kind of amalgamation was bidirectional, since on the one hand, because of Han-Tibetan intermarriages and the requirements of the environment, the Han people who lived in those areas for several generations integrated into the Tibetan ethnic group; on the other hand, these Han people added elements of Han culture to the local Tibetan culture, forming a situation in which "the Han people are not pure, and the Tibetans are not entirely Tibetan" (Xia Wu 2007), meaning that the cultural state in which Han and Tibetans mixed, substantially increasing the compatibility and intermediacy between local Tibetans and Han culture, as 
well as strengthening the connections and binding role of the Tibetan-Yi Corridor as the "Han-Tibetan border" between the Han and the Tibetans.

The large scale interaction and cultural amalgamation between the Han and the Tibetans caused by the major migration in the Qing dynasty and the Republican era is the most noticeable ethnic phenomenon in the Tibetan-Yi Corridor in modern times, not only furthering the breadth and depth of the blending and mixing of Han and Tibetan cultures in this region, but also transforming the Tibetan-Yi Corridor into a channel for ethnic exchanges from east to west, and what scholars call the "Han-Tibetan ethnic corridor"(Ren Xinjian, 2004).

\section{Conclusions}

There is no doubt that the main five ethnic flows in the history of the Tibetan-Yi Corridor outlined above have shaped the causes and process of today's ethnic structure of the Tibetan-Yi Corridor. From these five main ethnic flow trends, we can see that the ethnic activities throughout the history of the Tibetan-Yi Corridor presented the following features:

As an "ethnic region shaped by history," the formation process of the ethnic composition of the Tibetan-Yi Corridor was characterized by great openness and fluidity. We can surprisingly find that the ethnic groups or ethnic systems living in the corridor today have almost without exception migrated from surrounding territories. The ancestors of the main ethnic system in the corridor, the Tibeto-Burman group, came from the upper reaches of the Yellow River, and gradually started migrating towards the Tibetan-Yi Corridor in the Neolithic era, not only becoming the earliest settlers in that region, but also developing the largest ethnic system there. As we consider history, we see ethnic groups like the Di and Qiang continuously moving from the Gansu-Qinghai region towards the Tibetan-Yi Corridor: their arrival created pressure on those who had migrated there before, leading to new ethnic flows. Thus, up until the Tang dynasty, the Tibeto-Burman group's migration from north to south had been the main population flow in the Tibetan-Yi Corridor, while the "Fan-isation" of the northern part of the corridor, that is the formation of a population composed by "Tibetans in the north" and "Yi in the south" was the consequence of the eastward expansion of the western Tubo kingdom and the following gradual propagation and development to the east of Tibetan Buddhism. The Mongols migrated twice southward, once during the Yuan dynasty and the other in the late Ming and early Qing, upsetting the ethnic structure of the corridor, and not only restraining the northward expansion of the $\mathrm{Mu}$ chieftains, but also strengthening and expanding the scope of the "northern Tibetans," thus gradually consolidating and finalizing the structure of "Tibetans in the north" and "Yi in the south" of the corridor. During the Ming and Qing dynasties, up until the Republican era, Han people migrated in great numbers from east to west, entering the Tibetan-Yi Corridor, increasing the openness of the region from east to west, and transforming it into a channel of communication between Han and Tibetans. It is important to note that in the historical development of the Tibetan-Yi Corridor, external ethnic groups and their political forces mainly entered from the north, south 
and east, greatly influencing this area. Apart from the southward migration of the Tibeto-Burman group from the north, the Tubo, Mongolian and Han people respectively came from the west, north and east. The Tubo entering the Tibetan-Yi Corridor from the west was an organic component of their eastward expansion, while the Mongols entered the corridor as a part of their strategy of "first taking the south-western Fans, and then conquering China"; the Han arriving in big waves during the Qing dynasty and the Republican era were the consequence of the central government's efforts to strengthen operations in Tibet and the border areas. From this we can see that the formation of the ethnic composition of the Tibetan-Yi Corridor was closely interconnected with the greater structure of Chinese history and ethnic development; the incessant arrival of external people and political forces became an important driving force in the ethnic flows and evolution of the corridor. Thus, even though the Tibetan-Yi Corridor was an independent "ethnic region shaped by history," the historical process that shaped it was by no means isolated or closed, but it was characterized by great openness. In other words, as a corridor for ethnic migration, the fluidity of ethnic groups of the Tibetan-Yi Corridor was greatly connected to its openness, and it was thanks to its openness and the continuous mixing of its ethnic composition that brought out its fluidity and constant contacts, interactions and mixing between ethnic groups, which perhaps is an important feature and pattern of ethnic corridors.

The Tibetan-Yi Corridor is a transitional, linking channel for ethnic flows, and it acts as a border for the contact and exchanges between the Han and Tibetans and between the Yi and Tibetans. If something is called "corridor," it means that it is a place of transition and linking. As a corridor for ethnic migrations, the Tibetan-Yi Corridor clearly possesses these qualities, which is why Fei Xiaotong called it "the border area for the contact between Han and Tibetans and between Yi and Tibetans". The transition and connection belt between Tibetans and Han goes from east to west in the Tibetan-Yi Corridor, which is the channel for the contacts, exchanges and interactions between Han and Tibetans, as well as the region of mutual cultural influence and blending between these two groups. It was precisely because of this that some scholars have used the name "Han-Tibetan Corridor," adopting the angle of the relations between the Han and Tibetans. Looking from north to south, we see the structure of "Tibetans in the north" and "Yi in the south," which is the border of contact between the $\mathrm{Yi}$ and Tibetans: thus another feature of the corridor is the contacts and interactions between $\mathrm{Yi}$ and Tibetans (the term $\mathrm{Yi}$ is used in its wider meaning here, mainly indicating the Yi subgroup living in the southern part of the corridor). It is necessary to point out that, as the "border of contact between Han and Tibetans and between Yi and Tibetans," the Tibetan-Yi Corridor was shaped through a long-term, gradual historical process, and was the result of the interaction of multiple factors. For example, when it comes to the borders between the Yi and the Tibetans, even though the eastward expansion of the Tubo, the southward expedition of the Mongols, the expansion of the Mu chieftains and the northward migration of the $\mathrm{Yi}$ were all important factors leading to the formation of the "Tibetans to the north, Yi to the south" pattern, as well as the borders 
between Tibetans and Yi, the formation of the borders between Tibetans and $\mathrm{Yi}$ depended on clear ecological factors. It is not difficult to see that because the Tibetan people were mostly adapted to the ecological environment at high altitudes, this caused the border between Tibetans and $\mathrm{Yi}$ in the corridor to often be located at the intersection between the Tibetan-Yi plateau and the Yunnan-Guizhou plateau, therefore the border between the Tibetans and Yi was a cultural border as well as an ecological border. The border between Han and Tibetans was also characterized by similar circumstances, although the Han-Tibetan border was formed through many years of contacts and interactions between the two ethnic groups, the Han people living in the corridor were mainly concentrated in towns, along traffic routes, and in low altitude river valleys, and formed intertwined and adjacent relations with the local Tibetans who lived at high altitude pastures. Therefore, the ethnic "borders" in the corridor were often multi-dimensional and closely related to the ecological environment. Furthermore, it is necessary to note that the ethnic "borders" in the Tibetan-Yi Corridor were in fact not just a linear or geographic "border"; for the ethnic groups living in "border" areas, the most important features were the "connection links" that enabled the interactions and mutual cultural acceptance and tolerance between different ethnicities, as well as becoming reciprocally closer psychologically and emotionally. For instance, the Nakhi creation epic Chong ban chongze contains the legend that the ancestors of the Nakhi and the Tibetans were brothers, and the ancestor of the Tibetans was the older brother, while the ancestor of the Nakhi was the second-born. (Lijiang Working Group, Creation Myth, 1960) Another Nakhi epic, the Creation Myth also says that the ancestors of the Tibetans, Nakhi and Bai people were brothers: "The three brothers spoke three different languages, and were the ancestors of three ethnic groups. The eldest one was Tibetan, and he lived on the top; [...] the youngest one was Bai, and he lived below; [...] the second son was Nakhi, and he lived in the middle" (Xijiang Long 1991). In the Tibetan-Yi Corridor there were also legends of the Yi and Tibetans descending from the same ancestor (Guoyu Fang 1984, Li Shaoming, Chen Xianmin, 1991). This kind of legends reflects the "ethnic views" of the local people, as well as their unique understanding of ethnic "borders." Furthermore, from the Song dynasty large quantities of Han-grown tea started to be transported to the Tibetan region through the Tibetan-Yi Corridor, becoming an important economic link between the Han and the Tibetans. The heroic epic of the local Tibetan people, the Epic of King Gesar contains the following explanation of the exchanges between $\mathrm{Han}$ and $\mathrm{Ti}$ betans by King Gesar himself: "Are the goods produced by the Han and transported to Bo (the Tibetan area) things that we cannot produce here? Not at all, but it is necessary to connect the two people "(Quoted from: Institute of Geographic Sciences and Natural Resources Research, Chinese Academy of Science, Report on the Feasibility of the Tourism Development of the Old Tea-Horse Road in Qamdo County, Tibet) 2001). This represents the unique understanding of the Tibetan people living in the Tibetan-Yi Corridor toward "borders." Generally speaking, "border" has two main functions: one is to 
"divide," and the other is to "link." Since the borders between the Han and Tibetans, and Yi and Tibetans in the Tibetan-Yi Corridor were gradually formed through a long historical process of ethnic contacts and interactions, the ethnic groups living along these "borders" have been living to this day in an intertwined fashion, becoming inseparable from each other. Thus, in the eyes of the people living in the corridor, "border" is a way of "connecting" rather than "dividing," creating friendly relations and mutual identities: this is perhaps an important characteristics of the ethnic "borders" of the Tibetan-Yi Corridor.

The important historical role of the Tibetan-Yi Corridor in connecting the northern and southern ethnicities, and the nomadic and farming ethnicities.

By conducting an overview of the ethnic flows throughout the history of the Tibetan-Yi Corridor, we see that ancestors of the Tibeto-Burman group, the Di and Qiang, and the Mongols during the Yuan and Ming dynasties, all migrated south from the Gansu-Qinghai regions in the upper reaches of the Yellow River along the Tibetan-Yi Corridor; many of them built close relations with the local population when they reached the southern part of the Tibetan-Yi Corridor. Especially during the two times when the Mongols moved south towards the Tibetan-Yi Corridor, conquering and ruling a great part of the corridor, this led numerous Mongols to settle down in the region, mixing with the local population. Furthermore, the majority of the ancestors of the Hui people currently living in Yunnan arrived from the northwest and migrated southward through the Tibetan-Yi Corridor during the Yuan dynasty. (Ma Weiliang, 1999) Therefore, the Tibetan-Yi Corridor has been to a great extent a channel for the southward migration of Chinese northern ethnicities, playing an important historical role in creating links and exchanges between the northern and southern peoples.

Moreover, the exchanges between northern and southern ethnicities through the Tibetan-Yi Corridor also included to a great extent the contacts and exchanges between nomadic groups and farming peoples. The large scale arrival of the Mongols in the Tibetan-Yi Corridor and their close interactions and mixing with the local population is a typical example of this. Apart from the Mongols, other northern nomadic groups that arrived in the Tibetan-Yi Corridor include the Tokharians, the Qidan, and the Huihu (Zengqi Zhang, 2006, Meng Zhidong, 1995. ZengXianjiang, 2006). Archaeological findings also show that from the Neolithic era to the Bronze age there were many cultural elements from the northern grasslands entering the Tibetan-Yi Corridor (Hang Feng 1961, Tong Enzheng, 1998, Shen Xu, 2000).

Therefore, in a certain sense the Tibetan-Yi Corridor was also an important area for the contacts, exchanges and amalgamation between the northern nomadic people and the southern farming groups. Since topographically speaking the Tibetan-Yi Corridor is high in the north, low in the south, the northern part is located within the geographical scope of the Qinghai-Tibetan plateau, while the south is situated in the Yunnan-Guizhou plateau, the northern part is predominantly pastures, the middle part is a mixture of pastures and farmland, and the south is a mix of mountainous areas and farming areas. Therefore, the Tibetan-Yi Corridor is located at the geographical belt of transition from the 
northern grasslands to the southern mountainous and farming areas. These features provide the natural foundations for the northern nomadic people to migrate to the south in the Tibetan-Yi Corridor. This is also the reason why throughout history the northern nomadic people were able to migrate southward, creating close relations with the southern farming population, thereby leading the Tibetan-Yi Corridor to play an important historical role in connecting the northwestern peoples with the southwestern ones, as well as the nomadic groups with the farming groups.

\section{Funding}

National social science foundation of China(Funding number:10\&ZD110).

\section{Availability of data and materials}

The materials in this paper are all write in Chinese, which contains articles and classical books, the articles can be find in the data base of cnki(http://kns.cnki.net/kns/brief/default_result.aspx), as to the classical books, some of them can be find on the internet, and some of them can be find in the library of Sichuan university, the author has been written clearly where the materials can be find, in which book, or in which article.

\section{Authors' contributions}

The author read and approved the final manuscript.

\section{Author's information}

The author study the history of Khams for a long time, he upholds the scholar's neutrality, and try to show people the real history of the Khams by reading the material of people from the same period, and do a lot of field work to understand the people there more. The author does not a Tibet, but he has strong love for the Tibet people.

\section{Ethics approval and consent to participate}

Not applicable

\section{Competing interests}

The author declares that he has no competing interests.

\section{Publisher's Note}

Springer Nature remains neutral with regard to jurisdictional claims in published maps and institutional affiliations.

\section{References}

According to a 1939 survey, in Dartsendo"apart from a small number of people coming from the central regions of China, nine tenths of the local Han population came from mixed families, with a Han father and a Tibetan mother." See He Xiangfeng, "Xikang Travel Diary, "Border Public 
Theory, 1941 (1), Combined Issue No. 3-4. According to a survey, around 1938 the Han-Tibetan mixed-race people in Dawu county represented two thirds of the total population. See Zhao Liufang, "Images of Dawu,"Kangdao Monthly Bulletin, 1938, First issue.

According to history books and Hui genealogies and family records, waves of the ancestors of Hui people entered Yunnan at the beginning of the Yuan dynasty. See Ma Weiliang, A Historical and Cultural Study on the Hui Nationality in Yunnan, Yunnan University Press, 1999, pp.26-27.

According to population statistics on Xikang in the 1930s, the Mongolians living in Ningjing county (namely Jiangka) reached $60 \%$ of the total population, furthermore the percentage of Mongolians over the total population varied for each county: Ba'an, 1\%; Yanjing, 5\%; Kemai, 10\%; Zayü, 4\%; Gongxian, 65\%; Kham, 10\%; Enda, 5\%; Shuodu, 5\%; Lhari, 5\%; Taizhao, 2\%; Sêrxü, 2\%; Dengke, 10\%; Tongpu, 10\%; Dêgê, 5\%. See Yang Zhonghua, Xikang Minutes, The Commercial Press (1937), pp.244-247.

Anonymous, "Ethnic Groups of Xikang,"Sichuan Monthly Bulletin, 1936 (9), No. 4.The term "Xikang” in the text indicates Kang area after the establishment of the province of Xikang.

Ma Changshou, Di and Qiang, Shanghai People's Publishing House, 1984, pp.166-181.

Chengdu Municipal Cultural Relics and Archaeology Institute, Aba Tibetan and Qiang Autonomous Prefecture Cultural Relics Office, Report on the Trial Excavation at the Yingpanshan Relics Site in Mao County, Sichuan; Chengdu Provincial Cultural Relics and Archaeology, ed., Archaeological Findings in Chengdu, Science Press, 2002, pp.1-77.

Chen Chongwei, The Xikang Issue, Zhonghua Book Company, 1930, p.90.

Creation Myth, collected, translated and collated by the Lijiang Working Group for Popular Literature of Ethnic Groups in Yunnan, Yunnan People's Publishing House, 1960, pp.93-94.

Cultural Relics Administrative Committee of the Tibetan Autonomous Region, History Department of Sichuan University, Kham Karuo, Cultural Relics Press, 1985.

Fang Guoyu, History of the Yi People, Sichuan Publishing House, 1984, pp.562-563.

Guoyu, Fang. 1991. A study on the Mexie people. In Selected works on the south-western ethnic groups, ed. Li Shaoming and Chen Xianmin, 265. Sichuan University Press.

See Feng Hanji, "An Exploration of the Issue of the Ethnic Origin of the Cultural Relics Excavated in Jinning Mountain, Yunnan, "Archaeology, 1961 (9). Tong Enzheng, "On the Cultural Propagation Region of the Half-Moon Border from North-East to South-West in China)," Tong Enzheng, "Southern Civilisations, "Collected Essays by Tong Enzheng, Academic Series, Chongqing Publishing Group, 1998, pp.558603. ShenXu, "The Tibetan-Yi Corridor and South-West Asian Cultures,"Tibetan Studies, 2000 (2).

History of Liao, Volume 36 Military Records, Volume 46, A Hundred Officials. Officials of the Northern Vassal States.

History of Ming, Volume 311, Records of Sichuan Chieftains Part One. Jianchang, pp.8017-8018.

History of Yuan, Chapter 149,Legend of GuoBaoyu.

History of Yuan, Vol. 61, Geographic Records.

In the Map of Xikang, compiled in 1933-1934, Ren Naiqiang wrote: "The Tubo dynasty used its people to conquer the territories of the Wei, Rong, Mao, Wen, Li, Ya, and used the Qiang to attack the lands west of the Yellow River and Longyou, for nearly two hundred years. Since then, the Tubo were identified with the Tibetan people, and they were collectively known as Fan (Bo) people, or Tubote." He also noted that "Ancient records in China would refer to the Tibetan ethnic group in Xikang with just the character Fan." The chapter on Popular Customs in the Map of Xikang also uses the name "Fan people" when introducing the Tibetan people in Xikang. See Ren Naiqiang, Map of Xikang, Tibetan Ancient Books Publishing House, 2000, pp.222-224.

List of Officials of the Mu Clan in Lijiang (Vol. 1)), Historical Survey on the Nakhi Society), Nationalities Publishing House of Yunnan, 1983.

Ming emperors Yingzong and Xianzong repeatedly ordered the envoys to the Wusi Tibetans to pass through Sichuan instead of Hezhou. Afterwards, the routes of the envoys between the Han and the Tibetans also mainly passed through Sichuan, using it as a formal relay station. See Zhao Yi, "transportations between inland China and Tibet during the Ming dynasty), "Chinese Tibetology, 1992 (2).

New Book of Tang, Chapter 216, The Tubo Commentary.

Old Book of Tang, Chapter 196, The Tubo Commentary

On the issue of ethnic classification in China," Social Sciences of China,1980(1).

Pan Fasheng, "The Story of the Power Expansion of the Lijiang Mu Chieftains in Tibet,"Tibetan Studies, 1999 (2).

People at that time would say: "In general the Han along the passage to the Kang Tibetans, nine tenths of them were descendants of the soldiers stationed there. 1935. In Minutes of Qing's sending troops to big and small Jinchuan and Xikang Qinghai, ed. Wu Feibai, 163. Printed copy. 
Yu Qingyuan, "Weixi Chronicles,"Weixi Historical Materials 2, compiled by the Weixi Lisu Autonomous Prefecture Editorial Committee Bureau, 1994, p.12.

Quoted from: Institute of Geographic Sciences and Natural Resources Research, Chinese Academy of Science, Report on the Feasibility of the Tourism Development of the Old Tea-Horse Road in Qamdo County, Tibet), 2001, Printed copy, p.133.

See Yunnan Bureau for the Plan of the Publication of National Minority Ancient Books, ed., Guide for Souls (Vol. 1), Nationalities Publishing House of Yunnan, 1989. GuojiNingha, Ling Fuxiang ed., Collection of Translations of the Yi Language 'Guide for Souls'), Minzu University of China, 1993.

Li Shaoming, The South-Western Silk Roads and the Ethnic Corridor, History Department of Sichuan University, ed., Ancient Traffic and Cultures in South-Western China, Sichuan University Press, 1994, pp.37-38.

Shen Zhongchang, Li Fuhua, "A Preliminary Study of Han Cultural Elements in the Culture of Sarcophagus Burial,"Archaeology and Cultural Relics, 1983 (1).

Shi, Shuo, "Looking at the Migration of the Population of the Upper Reaches of the Yellow River to the Tibetan-Yi Corridor from the Perspective of Neolithic Cultures, "Journal of Southwest Nationalities College, 2008 (10).

Shuo, Shi. 1997. Tibetan Buddhism and the formation of the Tibetan ethnic group. Journal of Sichuan University 3.

Shi Shuo, Ethnic Origin of the Tibetan People and the AncientCivilisation of Eastern Tibet, Sichuan People's Publishing House, 2001, pp.54-65.

Shi Shuo, The Regional Classification of Neolithic Cultures in the Tibetan-Yi Corridor and their Relation to the Gansu-Qinghai Region, Cultural Studies in Bashu (Part III)), Bashu Books, 2006 (May).

Shi Shuo, "An Investigation on the Migration Routes of the Neolithic Population of the Upper Reaches of the Yellow River towards the Tibetan-Yi Corridor,"Journal of Southwest Nationalities College, 2009a (6).

Shuo, Shi. 2009b. The implication of the "xi" people as part of the south-western non-Han people during the Han dynasty: A discussion on the southward migration of the Shu people and their integration with the south-western non-Han population. Ethno-National Studies (6) Shi Shuo, "The South-Western non-Han People Shoubetween the Han and Jin Dynasties and their Relation with the Shu People),"Ethno-National Studies, 2011 (6).

Shi Shuo, The Tibetan-Yi Corridor: The Origin of Civilisation and Ethnic Origins, Sichuan People's Publishing House, 2009c, pp.63-70.

Shuzi, Huang. 1934. Surviving manuscript of the Mengxuan. Northwest Inland Exploration (2) No. 6.

Zhonghua Book Company. Wangshihejiaoshuijingzhu(The Four Divisions.Historical Classics. Commentary on the ShuiJingzhu), Zhonghua Book Company, 1978, 483. The Old Book of Tang. Geography Records under the government of the commander-in-chief of Annam quotes the Records of Annam: "Cochin was the most fertile region. There was a brave sovereign, with brave supporters. The Shu king sent thirty thousand soldiers to fight the brave sovereign, and was annihilated. The Shu king was the king of Anyang, who ruled over Cochin.'

Sichuan Provincial Cultural Relics and Archaeology Institute, Bureau of Cultural Affairs of the Garze Tibetan Autonomous Prefecture, A Presentation of the DanbaHan'eyi Excavation Site; Sichuan Provincial Cultural Relics and Archaeology Institute, ed., Collection of Archaeological Reports in Sichuan, Cultural Relics Press, 1998, p.74.

Sichuan Provincial Editing Group, Selected Works on Investigation Data and Archive Material on the History of the Yi People in Sichuan, Sichuan Academy of Social Sciences Press, 1987, pp. 87-89.

Zhou Taixuan, General History of Xikang. Industrial and Commercial History, p.37, Sichuan Provincial Archive Collection.

Ni Tuiji, Calendars of Yunnan, Vol. 10, Taipei Wenyuange Copy of the SikuQuanshu, 1986.

Vol. 22 Chronicles of Puliof the Anshun Government Records,Guizhou, cites the ancient record in Yi language Yi Book of Luogui, according to which "Mengzhe, the founder [ the ancestors of the Yi people] moved away from outside the land of the yaks and settled in Qiongzhilu."Brief History of the DebuPeople, (duplicate of the translation), translated by the GuizhouBijie Prefecture Yi Language Translation Unit. See also Meng Mo, "On the Question of the Origins of the Yi people,"The Ideological Front, 1980 (1).

Zhou Weizhou, "A Study on the Wenmo Army,"North-West Historical Data, 1982 (2).

Cheng Wenhai, "Stele on the Pacification of Yunann," Collection of Xuelou, Vol. 5, Taipei Wenyuange Copy of the SikuQuanshu, (Yuan) 1986.

Wu Xia, "A Study on the History of the Tibetan Society in Jiulong," in Li Shaoming, Liu Junbo, ed.,Ersu Tibetan Studies, Ethnic Publishing House, 2007, p.268. 
Xiaotong, Fei. 1982. An in-depth discussion of the issue of conducting ethnic research. Journal of SouthCentral University for Nationalities 3.

Xijiang, See Long. 1991. An investigation on the 'western fan' in the region of Liangshan and their origins, part 1. Tibetan Studies (1).

Zhao Xinyu, "The Punitive Expedition to the South of the Heshuote Tribe and its Influence on the Tibetan Areas in Sichuan and Yunnan,"Journal of Yunnan Minzu University, 2002 (3).

Yang Jiaming et al., Garze Ethnography, Contemporary China Publishing House, 1997, p. 277.

Zeng Yanshu, "Journal of an Official in Kangnan,"Kangdao Monthly Bulletin, 1943 (5), No. 6.

Yunnan Ethnic Minorities Socio-historical Survey Team, Chinese Academy of Social Sciences, ed., Brief History of the Yi People (First draft), Internal printing copy, 1963, p.118.

Zhang Zengqi, The State of Dian and Dian Culture, Yunnan Fine Arts Publishing House, pp.274-287. Meng Zhidong, A Study on the Descendants of the Khitans in Yunnan, China Social Sciences Press, 1995. ZengXianjiang, "A Study on the Origins and Historical Development of the Huo'erPeople in Northern Kang,"Ethno-National Studies, 2006 (5).

Liu Zhenggang, Tang Weihua, "Migration and Development of the Sichuan and Tibetan Area during the Qing Dynasty),"Tibetan Issues, 2002 (1). 